\title{
Efeito do alumínio em plantas de Pinhão-Manso (Jatropha curcas L.), cultivadas em solução nutritiva
}

\section{Effect of aluminum in plants of Jatropha curcas L. grown in nutritive solution}

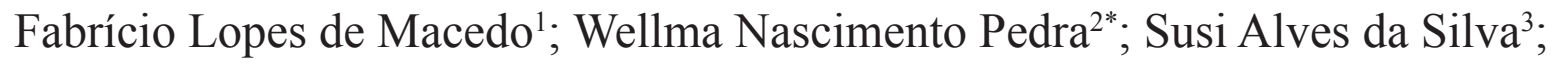 \\ Marcos Cabral de Vasconcellos Barreto ${ }^{4}$; Renata Silva-Mann ${ }^{5}$
}

\begin{abstract}
Resumo
Quantidades elevadas de alumínio $\left(\mathrm{Al}^{3+}\right)$ limitam o desenvolvimento de muitas espécies vegetais, inibindo o crescimento de suas raízes. O pinhão-manso (Jatropha curcas L.) tem sido estudado amplamente para a produção de biodiesel e, apesar de ser tolerante em solos com baixa fertilidade, as raízes do pinhão-manso não se desenvolvem em solos ácidos. Este trabalho teve como objetivo avaliar o desenvolvimento e a resposta fisiológica de diferentes acessos de pinhão-manso em doses variadas de alumínio. Plântulas obtidas de sementes provenientes de São Paulo, Minas Gerais e Bahia foram submetidas a estresse de alumínio, nas concentrações $0 ; 3,0 ; 4,5$ e $6,0 \mathrm{mg} \mathrm{L}^{-1} \mathrm{em}$ solução nutritiva, sendo avaliados os parâmetros: massa fresca da parte aérea (MFPA), massa fresca da raiz (MFR), massa seca da parte aérea (MSA), massa seca da raiz (MSR), comprimento inicial da raiz antes da submersão em solução (CIR) e crescimento final da raiz após submersão em solução (CFR). Para as variáveis MFPA, MFR e MSR, os acessos provenientes de Minas Gerais e Bahia apresentaram médias superiores em relação aos de São Paulo. As doses de alumínio diferiram para as variáveis MFR e CFR após submersão em solução, sendo que estes resultados ajustados à equações lineares descendentes. O crescimento da espécie é afetado pela presença de alumínio, mesmo para as menores doses e independe da procedência. As doses de alumínio afetam o crescimento das raízes para todas as procedências, sendo as doses superiores a $3 \mathrm{mg} \mathrm{L}^{-1}$, as mais prejudiciais ao crescimento.
\end{abstract}

Palavras-chave: Toxidez, alumínio, biodiesel

\begin{abstract}
High amounts of aluminum $\left(\mathrm{Al}^{3+)}\right.$ limit the development of many plant species, inhibiting the growth of roots. The physic nut (Jatropha curcas L.) has been studied extensively for the production of biodiesel and despite being tolerant to low fertility soils, the roots of physic nuts do not developed in acid soils. This study aimed to evaluate the development and physiological responses of different accessions of physic nuts in varying doses of aluminum. Seedlings obtained from seeds from São Paulo, Minas Gerais and Bahia, were subjected to aluminum stress at concentrations of $0,3.0,4.5$ and $6.0 \mathrm{mg} \mathrm{L}^{-1}$ in nutritive solution, with the following parameters: fresh matter of shoot (MFPA), fresh matter of root (MFR), dry matter
\end{abstract}

\footnotetext{
1 Eng ${ }^{\mathrm{o}} \mathrm{Agr}^{\mathrm{o}}$, Mestre em Agroecossistemas pela Universidade Federal de Sergipe. E-mail: fabriciolmacedo@hotmail.com

2 Bióloga, Mestre em Agroecossistemas pela Universidade Federal de Sergipe. E-mail: wellmabio@yahoo.com.br

3 Eng ${ }^{\circ}$ Agr $^{\circ}$, Mestre em Agroecossistemas pela Universidade Federal de Sergipe. E-mail: susi_agro@yahoo.com.br

4 Eng ${ }^{\mathrm{o}} \mathrm{Agr}^{\mathrm{o}}$, D.Sc., Professor do Dep. de Engenharia Agronômica, da Universidade Federal de Sergipe. E-mail: mbarreto@ufs.br

5 Eng ${ }^{\mathrm{o}}$ Agr $^{\mathrm{o}}$, D.Sc., Professora do Dep. de Engenharia Agronômica, da Universidade Federal de Sergipe. E-mail: rsmann@ sergipenet.com.br

Autor para correspondência
} 
of shoot (MSA), dry matter of root (MSR), initial length of root before submersion in solution (CIR) and growth of root end after submersion in solution (CFR). For variables MFPA, MFR and MSR, the access from Minas Gerais and Bahia presented higher relative mean different from São Paulo provenance at levels of 5\% and 1\% significance, respectively. The doses of aluminum showed significant differences for the variables MFR and CFR after submersion in solution, and those results are explained by descendants linear equations. Based on these results, it was noticed that the growth of the species is affected by the presence of aluminum, even considering small levels and independently of the provenance. The doses of aluminum affect root growth for all provenances, with doses above $3 \mathrm{mgL}^{-1}$, the most harmful to plant growth.

Key words: Toxicity, aluminum, biofuel

Um dos principais fatores que oferece restrições para os cultivos agrícolas nos trópicos está relacionado à acidez dos solos. Segundo Sousa e Lobato (2004) os solos brasileiros apresentam um nível de acidez considerável, com a presença de alumínio em níveis prejudiciais ao desenvolvimento dos vegetais, além de apresentarem teores reduzidos de cálcio e magnésio trocáveis, demonstrando com isso situações prejudiciais ao desenvolvimento das culturas.

O alumínio no solo apresenta uma maior atividade quando o $\mathrm{pH}$ do solo diminui abaixo de 5.5, ocasionando, consequentemente, uma maior potencialização dos efeitos fitotóxicos e insalubres desse elemento às culturas (SALET; ANGHINONI; KOCHHANN, 1999).

Os efeitos biológicos causados pela presença do alumínio se mostram mais evidentes nas raízes, devido a sua baixa mobilidade na planta (GIAVENO; MIRANDA-FILHO; FURLANI, 2001). A acidez do solo torna o alumínio disponível para atuar nos sistemas radiculares da maioria das culturas (TOMAR, 2001), já que o nível alto de acidez no solo resulta na dissolução de minerais de argila e óxidos de alumínio, ocasionando o aparecimento da forma trocável, sendo a disponibilidade deste elemento, para algumas espécies, um fator limitante na produtividade da cultura, em solos ácidos (BONATO et al., 2000). O acúmulo de alumínio ocorre, preferencialmente, no sistema radicular das plantas, reduzindo então seu crescimento e desenvolvimento, causando um aumento no diâmetro das raízes e diminuição do número de raízes laterais.

O alumínio no meio de crescimento influencia a absorção de elementos essenciais, como $\mathrm{P}, \mathrm{Ca} \mathrm{e}$ Mg (LÓPEZ-BUCIO et al., 2000). Diversos autores tem relatado como primeiros sintomas observados da toxicidade por alumínio, aqueles relacionados à diminuição do crescimento das raízes (GONZALES et al., 1976; ANIOL, 1984; OLIVEIRA; RENA, 1989).

A utilização de corretivos agrícolas na prática visa diminuir a acidez do solo mediante elevação do $\mathrm{pH}$, além de fornecer outras melhorias no solo como a diminuição da toxidez de Alumínio, Fe e $\mathrm{Mn}$; fornecimento de $\mathrm{Ca}$ e $\mathrm{Mg}$ ao solo; aumento da CTC dentre outras melhorias (MUNSON, 1982).

Incentivos governamentais recomendam algumas plantas para a produção de biodiesel, dentre elas está o pinhão-manso (Jatropha curcas L.), que tem se mostrado uma fonte promissora de matéria prima para a produção de óleo. O pinhão-manso é uma espécie oleaginosa, de fácil propagação e que pode apresentar relevante importância social e econômica para o Brasil (SILVA et al., 2009).

Esta planta pertence à família das Euforbiáceas, procedente da América do Sul, possivelmente com origem no Brasil, sendo praticamente em todas as regiões do país, adaptando-se em condições edafoclimáticas variáveis, propagando-se, sobretudo nos Estados do Nordeste, Goiás e Minas Gerais. Uma característica interessante da cultura 
é sua boa adaptação a solos de baixa fertilidade (EMBRAPA, 2006). Em estudos realizados por Arruda et al. (2004) foi constatado que em solos ácidos, com pH abaixo de 4,5 as raízes do pinhãomanso não se desenvolvem satisfatoriamente.

Devido a poucos dados referentes à nutrição, e especificamente sobre a tolerância de plantas de pinhão-manso ao alumínio, o trabalho foi conduzido visando a avaliação do desenvolvimento inicial de mudas desta espécie em doses variadas de alumínio por meio de solução nutritiva, visando à identificação de doses que alterem a morfologia da espécie, assim como a resposta diferencial de acessos procedentes de São Paulo, Bahia e Minas Gerais.

O experimento foi conduzido em condições de estufa agrícola no Departamento de Engenharia Agronômica da Universidade Federal de Sergipe. $\mathrm{O}$ delineamento experimental utilizado foi $\mathrm{o}$ inteiramente casualizado em esquema fatorial $3 \mathrm{x} 4$ (3 procedências e 4 doses de alumínio) com três repetições. Para a obtenção de mudas de pinhãomanso utilizou-se sementes de três Estados do
Brasil (São Paulo, Minas Gerais e Bahia), que foram colocadas para germinar utilizando-se tubetes cônico de 40 x $140 \mathrm{~cm}^{3}$.

Duas semanas após a germinação das sementes, as plântulas tiveram suas medidas iniciais avaliadas por meio do comprimento das raízes, após isso, as mesmas foram acondicionadas em recipientes plásticos com capacidade de $1 \mathrm{~L}$, providos de tampas perfuradas, permitindo a submersão das raízes na solução nutritiva para os diferentes tratamentos.

Os tratamentos fixos da solução foram constituídos de todos os nutrientes minerais, nas concentrações apresentadas na Tabela 1, cuja composição química é uma modificação daquela proposta por Furlani e Hanna (1984). O estresse de alumínio foi obtido por meio de adições de volumes conhecidos de uma solução contendo $3,0 \mathrm{mg} \mathrm{L}^{-1}$ de $\mathrm{Al}^{3+}$, preparada a partir de $\mathrm{AlK}\left(\mathrm{SO}_{4}\right)_{2} \cdot 12 \mathrm{H}_{2} 0$ $\left(52,77 \mathrm{~g} \mathrm{~L}^{-1}\right)$. Foram utilizadas soluções nutritivas completas $\left(\operatorname{sem~} \mathrm{Al}^{3+}\right)$ e soluções com concentrações de 3,0; 4,5 e 6,0 $\mathrm{mL} \mathrm{L}^{-1}$ de $\mathrm{Al}^{3+}$, com ajuste diário do $\mathrm{pH}$, pela adição de $\mathrm{HCl} 0,5 \mathrm{M}$, ou $\mathrm{NaOH} 0,5 \mathrm{M}$, para que o mesmo se mantivesse na faixa de $4 \pm 0,3$.

Tabela 1. Composição das soluções estoques e da solução nutritiva usadas para avaliar genótipos de pinhão manso em função das concentrações de alumínio.

\begin{tabular}{|c|c|c|c|}
\hline \multirow[t]{2}{*}{ Solução } & \multicolumn{2}{|c|}{ Solução Estoque } & \multirow{2}{*}{$\begin{array}{l}\text { Volume utilizado (ml), para obtenção } \\
\text { de 1L da solução nutritiva }\end{array}$} \\
\hline & Componente & Concentração (g/L) & \\
\hline 1. & $\mathrm{Mg}\left(\mathrm{NO}_{3}\right)_{2} \cdot 6 \mathrm{H}_{2} \mathrm{O}$ & 142,4 & 0,28 \\
\hline 2. & $\mathrm{KH}_{2} \mathrm{PO}_{4}$ & 17,6 & 0,05 \\
\hline \multirow[t]{2}{*}{3.} & $\mathrm{Ca}\left(\mathrm{NO}_{3}\right)_{2} \cdot 4 \mathrm{H}_{2} \mathrm{O}$ & 270,0 & 0,66 \\
\hline & $\mathrm{NH}_{4} \mathrm{NO}_{3}$ & 33,8 & \\
\hline \multirow[t]{2}{*}{4.} & $\mathrm{FeSO}_{4} \cdot 7 \mathrm{H}_{2} \mathrm{O}$ & 24,9 & 0,33 \\
\hline & $\mathrm{Na}_{2}{ }_{\mathrm{EDTA}}^{2}$ & 29,6 & \\
\hline \multirow[t]{3}{*}{5.} & $\mathrm{KCl}$ & 18,6 & 0,46 \\
\hline & $\mathrm{KNO}_{3}$ & 24,6 & \\
\hline & $\mathrm{K}_{2} \mathrm{SO}_{4}^{3}$ & 44,0 & \\
\hline \multirow[t]{5}{*}{6} & $\mathrm{MnCl}_{2} \cdot 4 \mathrm{H}_{2} 0$ & 2,34 & 0,33 \\
\hline & $\mathrm{H}_{3} \mathrm{BO}_{3}$ & 2,04 & \\
\hline & $\mathrm{CuSO}_{4} \cdot 5 \mathrm{H}_{2} 0$ & 0,20 & \\
\hline & $\mathrm{ZnSO}_{4} \cdot 7 \mathrm{H}_{2} \mathrm{O}$ & 1,41 & \\
\hline & $\mathrm{Na}_{2} \mathrm{MoO}_{4} \cdot 2 \mathrm{H}_{2} 0$ & 0,26 & \\
\hline 7. & $\mathrm{AlK}\left(\mathrm{SO}_{4}\right)_{2} \cdot 12 \mathrm{H}_{2} 0$ & 52,77 & \\
\hline
\end{tabular}

Fonte: Furlani \& Hanna (1984). 
As plântulas ficaram sob a influência das diferentes doses de alumínio durante sete dias. Após esse período, foi medido novamente o sistema radicular e pesado a parte aérea das plântulas, após essas medições, toda estrutura vegetal das plântulas foram separadas e acondicionadas em sacos de papel e submetidos à secagem em estufa, com ventilação forçada, por um período de $48 \mathrm{~h}$ a $75^{\circ} \mathrm{C}$.

Os parâmetros avaliados ao final do processo foram: massa fresca da parte aérea (MFPA), massa fresca da raiz (MFR), massa seca da parte aérea (MSPA), massa seca da raiz (MSR), comprimento inicial da raiz (antes da submersão em solução) (CIR) e crescimento final da raiz (após a submersão na solução) (CFR).

A análise de variância foi realizada com o programa estatístico SISVAR $^{\circledR} \quad$ (FERREIRA,
2003), as médias comparadas pelo teste de Scott-

Knott a 5\% de probabilidade, e influência das doses verificadas por meio de regressão polinomial.

Após a análise dos resultados observou-se que a massa fresca da raiz (MFR) variou entre os tratamentos para as diferentes procedências $(\mathrm{p}<0,01)$, sendo que o material com procedências MG e BA não diferiram entre si e apresentaram médias superiores ao material com procedência SP, diferindo deste (Tabela 2). Por meio da avaliação da analise de regressão para a variável MFR para os tratamentos doses de $\mathrm{Al}^{3+}$ (Figura 1) observouse que a testemunha diferiu significativamente dos demais com média superior. Observou-se para as doses de 3,0 e 4,5 $\mathrm{mg} \mathrm{L}^{-1}$ valores superiores a dose $6,0 \mathrm{mg} \mathrm{L}^{-1}$ de $\mathrm{Al}^{3+}$. Visualiza-se um decréscimo da MFR com o aumento das doses de $\mathrm{Al}^{3+}$.

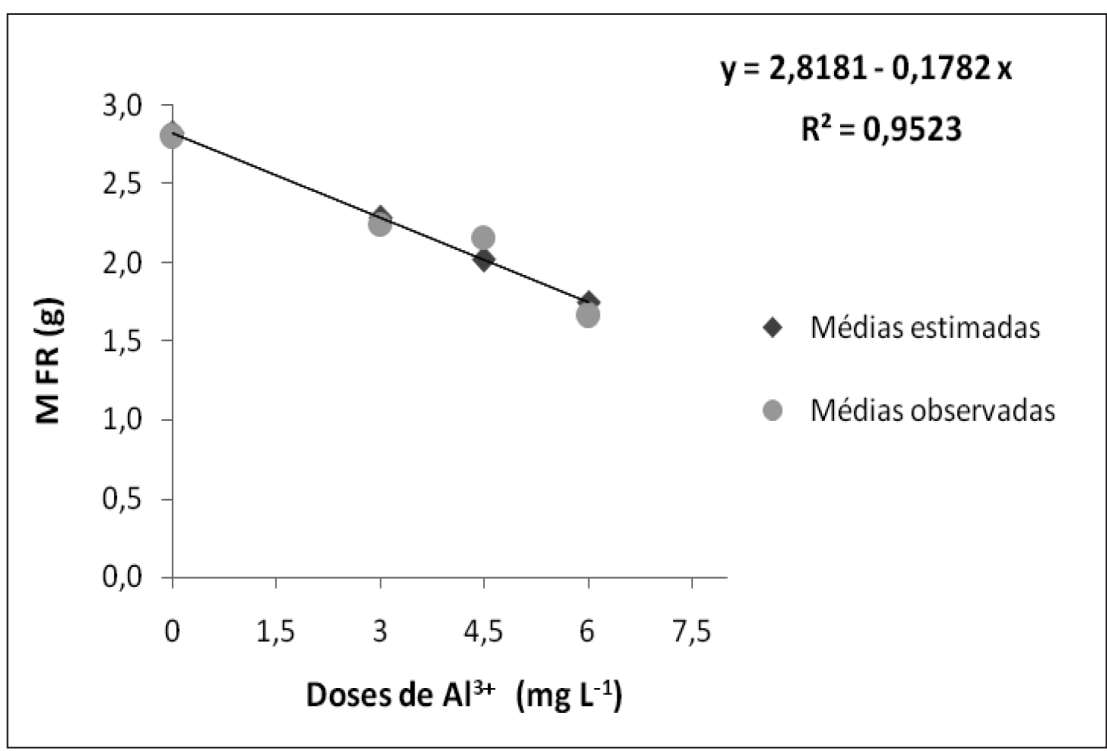

Figura 1. Massa fresca da raiz (MFR) das plantas de pinhão manso em resposta a diferentes doses de alumínio $\left(\mathrm{Al}^{3+}\right)$.

Para a massa fresca da parte aérea (MFPA) e massa seca da raiz (MSR) pode-se observar diferenças para os tratamentos $\mathrm{p}<0,05$ e $\mathrm{p}<0,01$, sendo os acessos procedentes de Minas Gerais e da Bahia os que apresentaram médias superiores àquelas apresentadas pelo acesso procedente de São Paulo (Tabela 2).
Para a variável crescimento final da raiz (CFR) observou-se diferenças significativas $(p<0,05)$ para as doses utilizadas, sendo observada uma redução linear com o aumento das doses (Figura 2). A maior média foi observada para o tratamento testemunha. 
Tabela 2. Médias obtidas da massa fresca da raiz (MFR), massa fresca da parte aérea (MFPA) e massa seca da raiz (MSR) para as diferentes procedências de pinhão manso.

\begin{tabular}{cccc}
\hline Procedência & MFR - Médias & MFPA - Médias & MSR - Médias \\
\hline S.P. & $1,696667 \mathrm{~b}$ & $7,280833 \mathrm{~b}$ & $0,121667 \mathrm{~b}$ \\
M.G. & $2,318333 \mathrm{a}$ & $8,509167 \mathrm{a}$ & $0,156667 \mathrm{a}$ \\
B.A. & $2,635000 \mathrm{a}$ & $8,570833 \mathrm{a}$ & $0,188333 \mathrm{a}$ \\
\hline CV $(\%)$ & 23.79 & 16.25 & 24.78 \\
\hline
\end{tabular}

Médias seguidas por letra igual não diferem entre si pelo teste Scott-Knott, a 5\% de probabilidade.

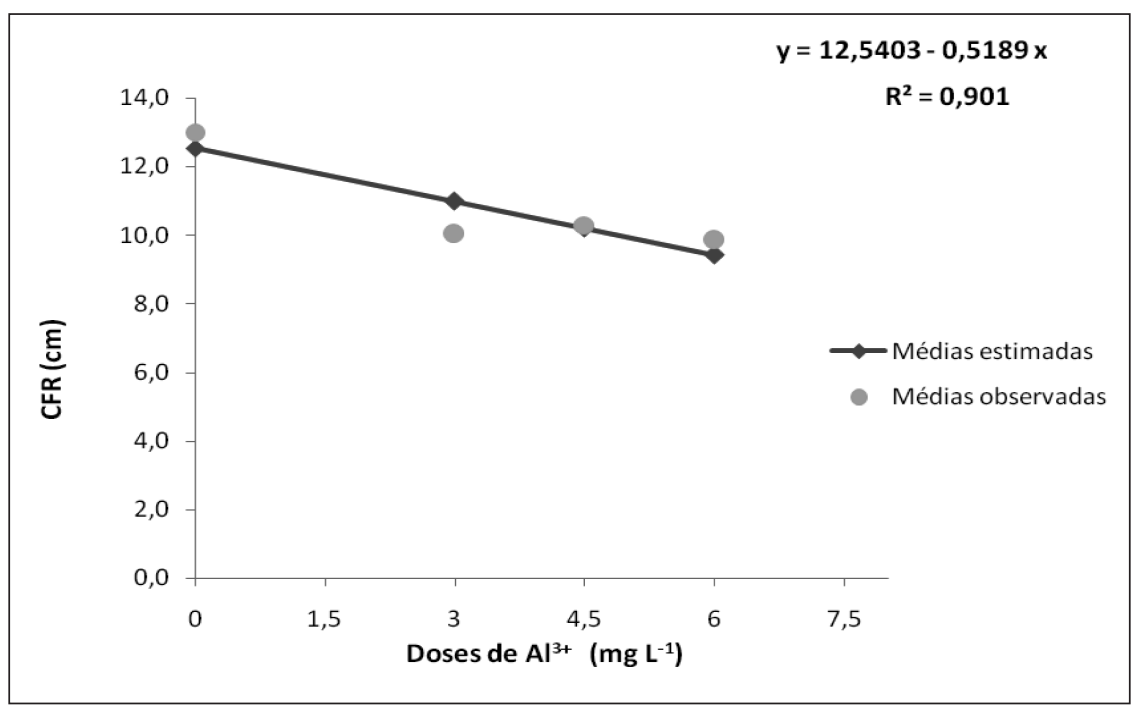

Figura 2. Crescimento final da raiz (CFR) de plantas de Pinhão manso em resposta a diferentes doses de alumínio $\left(\mathrm{Al}^{3+}\right)$.

Comportamento semelhante têm sido observado em espécies como mamoneira (Lima et al., 2007), sendo este um comportamento preditivo, no entanto, há que se considerar que o desenvolvimento de plântulas de uma espécie como o pinhão-manso deve ser testada visando a definição de condições de manejo para a cultura.

Para as variáveis massa seca de parte aérea (MSPA) e crescimento inicial da raiz (CIR) não observou-se diferença significativa $(p>0,05)$ para as diferentes procedências. Passos et al. (2008) não observaram interação genótipo $\mathrm{x}$ dose na avaliação do comprimento da raiz principal e retomada do crescimento da raiz principal no estudo da tolerância de genótipos de mamoneira quanto à tolerância ao alumínio.
Há que se considerar, que não somente as mensurações podem expressar as alterações promovidas pela toxidez de alumínio, uma vez que os danos causados nas raízes pela ação do $\mathrm{Al}^{3+}$ foram, facilmente, visualizados caracterizado pelo escurecimento nos pontos de crescimento das raízes, concordando com as descrições de Oliveira (2002) e Sánchez-Chacon et al. (2001), que consideraram doses de 15 ppm como suficientes para diferenciar cultivares sensíveis e tolerantes ao se avaliar o crescimento de raiz. Em experimento realizado, foi verificado que apenas $3 \mathrm{mg} \mathrm{L}^{-1}$ de $\mathrm{AL}^{3+}$ foram suficientes para causar esse efeito de escurecimento nas raízes de pinhão-manso.

Para espécies de caráter perene, o que se assemelha muito ao caráter do pinhão-mano, que 
pode ser produtiva por até 40 anos, jovens de seringueira Hevea brasiliensis [Willd. Ex. Adr. de Juss.) Muell.-Arg.] cultivadas na presença de alumínio, cádmio, níquel e zinco, Cupertino (2006) verificou-se,uma inibição do crescimento da raiz e da parte aérea, redução na produção de massa seca em todos os órgãos da planta, queda na taxa de fotossíntese e na resistência estomática, elevação na taxa de transpiração e redução na atividade da redutase do nitrato. Além disso, houve efeito significativo para os teores dos macronutrientes nas plantas avaliadas.

Os acessos procedentes da Bahia e de Minas Gerais são menos susceptíveis à toxidez de alumínio, quando comparados com os provenientes de São Paulo.

As doses de alumínio afetam o crescimento das raízes para todas as procedências, sendo as doses superiores a $3 \mathrm{mg} \mathrm{L}^{-1}$, as mais prejudiciais ao crescimento da planta.

\section{Referências}

ANIOL, A. Induction of aluminum tolerance in wheat seedlings by low closes of aluminum in the nutrient solution. Plant Physiology, Washington, v. 75, n. 2, p. 551-555, 1984.

ARRUDA, F. P. de; BELTRÃO, N. E. de M.; ANDRADE, A. P. de; PEREIRA, W. E.; SEVERINO, L. S. Cultivo de Pinhão manso (Jatropha curcas L.) como alternativa para o semi-árido Nordestino. Revista Brasileira de Oleaginosas e Fibrosas, Campina Grande, v. 8, n. 1, p. 789-799, 2004.

BONATO, C. M.; CAMBRAIA, J.; SANT'ANNA, V.; VENEGAS, H. A. Efeito do alumínio sobre a absorção, a partição e a utilização de enxofre em sorgo. Revista Brasileira de Fisiologia Vegetal, Campinas, v.1 2, n. 1, p. 17-24, 2000.

CUPERTINO, I .C. F. S. Respostas morfofisiológicas e nutricionais de plantas jovens de seringueira Hevea brasiliensis [Willd. Ex. Adr. de Juss.) Muell.-Arg.] cultivadas na presença de alumínio, cádmio, níquel e zinco. 2006. Tese (Doutorado em Agronomia) Universidade Federal de Lavras, Lavras.
EMPRESA BRASILEIRA DE PESQUISA AGROPECUÁRIA - EMBRAPA. Pinhão manso, opção de biodiesel no semi-árido. 2006. Disponível em: $\quad<$ http://www.embrapa.gov. br//noticias/banco_de_noticias/folder.2006/ setembro/foldernoticia2006-09-06.0580910313/ noticia.2006-09-25.0180290792/mostra_noticia>. Acesso em: 31 de mar. 2009.

FERREIRA, D. F. Sisvar versão 4.2. DEX/UFLA, 2003. 1 CD-ROM

FURLANI, P. R.; HANNA, L. G. Avaliação da tolerância de plantas de arroz e milho ao alumínio em solução nutritiva. Revista Brasileira de Ciência do Solo, Campinas, v. 8, n. 2, p. 205-208, 1984.

GIAVENO, G. D.; MIRANDA-FILHO, J. B.; FURLANI, P. R. Inheritance of aluminum tolerance in maize (Zea mays L.). Journal of Genetics \& Breeding, Rome, v. 55, n. 1, p.5 1-55, 2001.

GONZALES, E.; WOLF, J. M.; SOARES, M. W. V.; GALENO, E. Relações entre toxidez de alumínio, desenvolvimento de raízes, absorção de água e produção de milho no oxisol (latosol vermelho escuro) do Distrito Federal. Ciência e Cultura, Brasília, v. 28, n. 2, p. 181182, 1976.

LIMA, R. L. S.; SEVERINO, L. S.; CAZZETA, J. O.; SAMPAIO, L. R.; LEÃO, A. B.; SOFIATTI, V. ; BELTRÃO, N. E. de M.; FREIRE, M. A. O.; SILVA, L. V. B. D. Tolerância de genótipos de mamoneira ao alumínio. In: CONGRESSO DA REDE BRASILEIRA DE TECNOLOGIA DE BIODIESEL, 2., 2007, Brasília. Anais... Brasília: DF: MCT/ABIPTI, 2007.

LÓPEZ-BUCIO, L.; NETO JACOBO, M. F.; RAMIREZRODRIGUES, V.; HERRARA-ESTELLA, L. Organic acids metabolism in plants: from adaptive physiology to transgenic varieties for cultivation in extreme soils. Plant Science, Limerick, v. 160, n. 1, p. 1-13, 2000.

MUNSON, R. D. Potassium, calcium and magnesium in the tropics and subtropics. International Fertilizers Development Cente, 1982. 62 p. (Technical bulletin, $\mathrm{T}-23)$.

OLIVEIRA, L. E. M de; RENA, A. B. Influência do alumínio sobre o comportamento nutricional de cultivares de mandioca em solução nutritiva. Pesquisa Agropecuária Brasileira, Brasília, v. 24, n. 9, p. 11191230, 1989.

OLIVEIRA, P. H. de. Herança genética e mapeamento molecular da tolerância à toxidade do alumínio em Aveia (Avena sativa L.). 2002. Tese (Doutorado em Fitotecnia) 
- Faculdade de Agronomia. Universidade Federal do Rio Grande do Sul, Porto Alegre.

PASSOS, A. R.; SILVA, S. A.; CARVALHO, F. I. F. de.; HARTWIG, I.; CRESTANI, M.; CANÇADO, E. dos S. Tolerância de cultivares de mamoneira à toxicidade de alumínio em solução nutritiva. In: CONGRESSO BRASILEIRO DE MAMONA ENERGIA E RICINOQUÍMICA, 3., 2008, Salvador. Anais... Salvador: SEAGRI, 2008.

SALET, R. L.; ANGHINONI, I.; KOCHHANN, R. A. Atividade do alumínio na solução do solo do sistema plantio direto. Revista Cientifica da UNICRUZ, Cruz Alta, v. 1, n. 1, p. 9-13, 1999.

SÁNCHEZ-CHACÓN, C. D.; CARVALHO, F. I. F.; SILVA, J. A. G.; BARBIERI, R. L.; KUREK, A.; MARCHIORO, V. S. Reação de cultivares elite de aveia quanto à toxicidade do alumínio em cultivo hidropônico. In: REUNIÃO DA COMISSÃO BRASILEIRA DE PESQUISA DE AVEIA, 21., 2001, Lajes. Anais... Lajes: Universidade do Estado de Santa Catarina, UDESC, 2001. p. 90-91.

SILVA, E. N.; SILVEIRA, J. A. G.; FERNANDES, C. R. R.; DUTRA, A. T. B.; ARAGÃO, R. M. de. Acúmulo de íons e crescimento de pinhão-manso sob diferentes níveis de salinidade. Revista Ciência Agronômica, Fortaleza, v. 40, n. 2, p. 240-246, 2009.

SOUSA, D. M.; LOBATO, E. Cerrado: correção do solo e adubação. 2. ed. Brasília: Embrapa Informação Tecnológica, 2004. 416 p.

TOMAR, J. B. Rainfed upland rice-problems and prospects. Journal of Maharashtra Agricultural Universities, Bombay, v. 26, n. 1, p. 81-87, 2001. 
